\title{
LA PRESENCIA DE IRÁN EN AMÉRICA LATINA A TRAVÉS DE SU INFLUENCIA EN LOS PAÍSES DEL ALBA*
}

\author{
THE PRESEN CE OF IRAN IN LATIN AMERICA THROUGH \\ ITS IN FLUEN CE ON THE ALBA COUNTRIES
}

\section{ISAAC CARO** E ISABEL RODRÍGUEZ***}

\section{RESUMEN}

En este artículo examinamos la presencia de Irán en América Latina a través de sus conexiones con tres países miembros de la Alternativa Bolivariana para América Latina y el Caribe (ALBA) -Venezuela, Nicaragua, Bolivia- y un observador de esta agrupación -Ecuador-. Postulamos que estas relaciones no se limitan al ámbito económico, sino que incorporan también los campos político, cultural y militar. Se trata de una relación estratégica, de la cual ambos lados pretenden sacar ventajas políticas, buscando opacar el aislamiento internacional de Irán, por una parte, y presentarse como una plataforma de oposición a los Estados Unidos, por otra, enmarcada en un encuentro sur-sur.

Palabras clave: Irán, islam, América Latina, religión.

\section{ABSTRACT}

In this paper, we examine the presence of Iran in Latin America through its connections with three countries that are members of the "Alternativa Bolivariana para América Latina y el Caribe" (ALBA) -Venezuela, Nicaragua, Bolivia- and one that is an observer of this association -Ecuador-. We argue that these relations cover not only the eco-

* Este artículo es parte del Proyecto FONDECYT No 1080024.

** Doctor en Estudios Americanos, Magíster en Estudios Sociales y Políticos Latinoamericanos. Académico del Departamento de Ciencia Política y Relaciones Internacionales de la Universidad Alberto Hurtado y del Instituto de Estudios Internacionales de la Universidad Arturo Prat.E-mail: icaro@uahurtado.cl

*** Doctora $\odot$ en Ciencia Política y Sociología, Magíster en Estudios Internacionales. Académica del Departamento de Ciencia Política y Relaciones Internacionales de la Universidad Alberto Hurtado. E-mail: irodrigu@uahurtado.cl 
nomic level, but incorporate also the political, cultural and military levels. This is a strategic relationship in which both sides seek political advantages. On one hand, Iran seeks to overcome its international isolation and present itself as a platform of opposition to the United States; on the other hand, framing the latter in the context of SouthSouth relations.

Keywords: Iran, Islam, Latin America, religion.

Recibido: 05.03.2009. Aprobado: 06.07.2009.

\section{INTRODUCCIÓN}

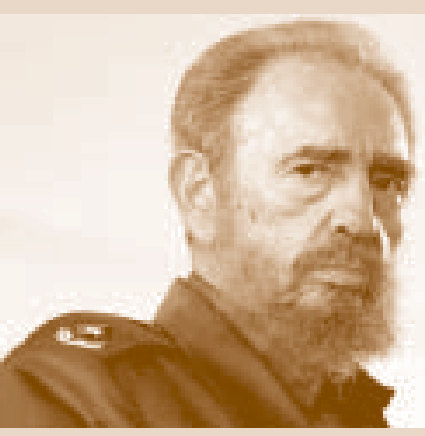

F. Castro

$\mathrm{E}$ N DICIEMBRE de 2004, los presidentes de Cuba, Fidel Castro, y Venezuela, Hugo Chávez, suscribieron en La Habana la denominada "Alternativa Bolivariana para América Latina y el Caribe", conocida como ALBA, con el objetivo de impulsar un proceso de integración regional propiamente latinoamericano como contrapartida al Área de Libre Comercio de las Américas (ALCA), impulsado por Estados Unidos desde diciembre de 1994. El ALBA ha logrado ampliarse a partir del año 2005 a razón de dos procesos que se refuerzan mutuamente: por una parte, el desarrollo de un esquema de integración regional al margen de cualquier participación de Estados Unidos, y por otra parte, el resultado de las elecciones ganadas por gobiernos simpatizantes a los regímenes de Cuba y Venezuela, como son Bolivia, Ecuador y Nicaragua.

La formación del ALBA responde, en gran medida, a un proceso promovido por el gobierno de Chávez, de fortalecer una agenda política, económica y cultural, que sea contraria a la de Estados Unidos, y que busque alternativas al modelo neoliberal que impera en otros países latinoamericanos. En el marco de esta oposición a Estados Unidos y de una diversificación de las relaciones latinoamericanas, los países del ALBA han buscado ampliar sus vínculos con potencias extrarregionales como Rusia y China, y, muy particularmente, con Irán, quien durante 2007, en el marco de la Cumbre del Movimiento de Países No Alineados, solicitó formalmente su incorporación como miembro observador al ALBA.

En cuanto a su ampliación, la incorporación de Bolivia se produce tras la victoria del presidente Evo Morales (2005), y la de Nicaragua, luego de la asunción del presidente Daniel Ortega (2006). Después de la llegada al poder del presidente Rafael Correa en Ecuador (2007), este país decide integrarse no como miembro pleno, sino como miembro observador. El ALBA ha logrado integrar también a otros estados, especialmente pertenecientes a la Comunidad Caribeña del CARICOM, como Antigua y Barbuda, Do- 
minica, San Vicente y Las Granadinas, que mantienen lazos comerciales con Venezuela, sobre todo en el campo del sector petrolero. En agosto de 2008 se incorpora como miembro pleno Honduras, con lo cual el ALBA agrupa ahora a estados del Caribe, de Sudamérica y de Centroamérica.

Si revisamos algunos antecedentes de las relaciones Irán-ALBA previos al 2004, hay varios aspectos a destacar. En primer lugar, están los vínculos que este país mantiene con Venezuela y Ecuador en el contexto de la Organización de Países Exportadores de Petróleo (OPEP), creada en Viena en el año 1960, y de la cual tanto Venezuela como Irán son miembros fundadores. Ecuador fue integrante entre 1973 y 1993, para volver a la OPEP en 1997. La presencia de Irán en la región está marcada también por otro antecedente de consideración: una vez ocurrida la revolución islámica en ese país (1979), Cuba se convierte en el primer aliado de Irán en la región, ambos países establecen relaciones diplomáticas ese año, y mantienen una agenda contraria a Estados Unidos, que tiene algunas coincidencias en la medida que ambos se enfrentan a las sanciones impuestas por Washington. También Irán y Nicaragua mantienen una alianza estratégica y antiestadounidense entre 1979 y 1990, con motivo del triunfo sandinista en este último país.

El presidente Muhamad Jatami, quien gobernó Irán entre 1997 y 2005, representaba una línea más bien moderada y reformista de la revolución islámica, partidario de hacer varios cambios aperturistas, lo que motivó que los sectores más duros del país, encabezados por Mahmud Ahmadinejad, se le opusieran vehementemente. Durante la administración de Jatami, la participación de Irán en América Latina se dio preferentemente en el marco de la OPEP, del Grupo de los 15 (G15) y de las relaciones bilaterales con Venezuela, sin llegar a mantener una alianza estratégica tan importante como la que consolidará su sucesor.

La llegada al poder de Hugo Chávez, la victoria de gobiernos izquierdistas como los ya mencionados, y la asunción del presidente Mahmud Ahmadineyad en agosto de 2005, son factores todos que inauguraron un ambiente propicio para una mayor presencia de Irán en la región latinoamericana, especialmente con los países que son miembros o simpatizantes del ALBA, presencia que va más allá de los ámbitos energéticos, comerciales y económicos.

La asunción de Ahmadineyad a la presidencia iraní impone una línea dura, partidaria de acabar con las reformas al interior de Irán, pronunciamientos reiterativos sobre la negación del holocausto, llamados a la destrucción de Israel y consolidación de un discurso abiertamente antiestado-

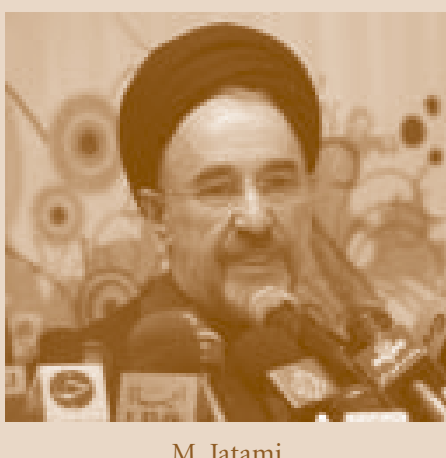

M. Jatami 


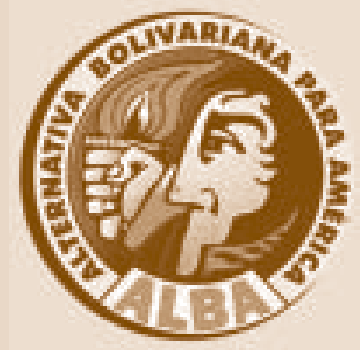

unidense, elementos que concuerdan con algunos pronunciamientos del presidente Chávez y de otros mandatarios afines al monarca bolivariano. Además, el nuevo presidente iraní consolida el programa de energía nuclear, sustentado en el enriquecimiento de uranio, lo que le permitiría tener armas nucleares en un plazo menor a cinco años, según autoridades de la Agencia Internacional para la Energía Atómica.

La presencia iraní se ha manifestado en el aumento del intercambio comercial y de inversiones con los países del ALBA, así como en las visitas del presidente Ahmadinejad a la región, las que han sido respondidas por sus homólogos latinoamericanos. A partir del 2006, el presidente iraní ha hecho tres visitas a la región, firmando acuerdos con estos países y abriendo embajadas en varios de ellos, como se verá más adelante. El año 2007 Irán anunció la planificación de nuevos proyectos en los sectores petrolero, industrial y financiero para cuatro países sudamericanos, tres de los cuales pertenecen al ALBA (Bolivia, Venezuela y Nicaragua).

Por su parte, el año 2008, autoridades iraníes señalaron que la ampliación de sus relaciones con los estados "latinoamericanos revolucionarios" era una prioridad de su política exterior. Teherán reiteró su disposición a consolidar la cooperación con los países del ALBA, incluidos Cuba, Bolivia, Ecuador y Honduras, al tiempo que muchos de éstos hicieron anuncios apoyando el programa de energía nuclear llevado a cabo por Teherán.

En este artículo examinaremos la presencia de Irán en América Latina a través de sus conexiones con tres países miembros del ALBA -Venezuela, Nicaragua, Bolivia-y un observador de esta agrupación -Ecuador-, postulando que ésta no se limita al ámbito económico, sino que incorpora también los campos político, cultural y militar. Se trata de una relación estratégica, de la cual ambos lados pretenden sacar provecho político, buscando opacar el aislamiento internacional de Irán, por una parte, y presentarse como una plataforma de oposición a Estados Unidos, por otra, enmarcada en un encuentro Sur-Sur. Un modelo islamista chíta y otro sustentado en el socialismo bolivariano se encuentran para dar respuestas a los desafíos señalados.

\section{IRÁN Y VENEZUELA: DE SOCIOS ENERGÉTICOS A ALIANZA ESTRATÉGICA}

Hasta la revolución islámica de 1979, Irán y Venezuela eran aliados de Estados Unidos. El régimen del sha Reza Pahlavi mantenía una plataforma de modernización pro occidental y pro estadounidense, seriamente compro- 
metido en su alianza con Israel y con Estados Unidos, con una importante presencia económica y militar de Washington (Arjomand, 1986). En cuanto a Venezuela, los gobiernos democráticos, que surgen tras la dictadura militar (1948-1958), van a ser un indicador de estabilidad regional, al tiempo que mantendrán significativas relaciones políticas y militares con Washington (Romero, 2006). Con la revolución islamista encabezada por el Ayatola Khomeini (1979) y, más tarde, con la llegada al poder de Hugo Chávez (1999), ambos estados pasan a ser decisivos opositores a Estados Unidos.

En el contexto de participación de Venezuela en la OPEP se pueden entender en parte los lazos que ha mantenido Caracas con países del Medio Oriente, y en particular con Irán, desde la fundación de esta organización en 1960. En agosto de 2000, y desafiando las sanciones impuestas por la ONU a Irak, el presidente Chávez visitó Bagdad, reuniéndose con su par Saddam Hussein. La caída de Hussein, tras la invasión de Estados Unidos en marzo de 2004, ha dejado a Irán como principal socio comercial y político de Venezuela en el marco de la OPEP.

Irán y Venezuela corresponden al segundo y cuarto productores de la OPEP, produciendo en forma conjunta el $9 \%$ mundial. Ambos países han influido para que Ecuador reingrese a la organización, en tanto que Sudán y Bolivia, que no son miembros de la OPEP, tienen concepciones estratégicas en materia energética similares a las de Chávez y Ahmadineyad. Las mayores reservas de hidrocarburos del mundo se encuentran en la Faja del Orinoco, donde Irán y Venezuela se han unido para crear una compañía petrolera internacional (Malamud, García, 2007).

Si bien es cierto que no se pueden desestimar los nexos que Venezuela ha logrado con el mundo árabe musulmán y, en especial con Irán, en el contexto de la organización exportadora de petróleo, hay que señalar que estas relaciones han superado ampliamente el ámbito de esta organización exportadora, motivando un distanciamiento con Washington, así como una preocupación por parte de Estados Unidos, de Israel y de las comunidades judías de toda la región.

¿Cómo se explican las relaciones bilaterales entre Venezuela e Irán más allá del ámbito de la OPEP? La llegada de Chávez al poder en 1999 y su proyecto de llevar a cabo una "revolución bolivariana", implicó la búsqueda de nuevos aliados, que contrarrestaran la presencia de Estados Unidos en la región. Irán, que había sido declarado como "país paria" por el gobierno de Washington, se presentaba como un aliado interesante para Caracas, con quien compartía ya una relación comercial importante en el marco de la OPEP.

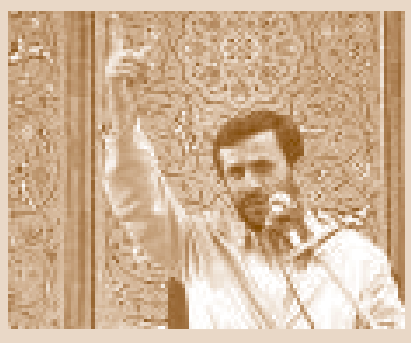

M. Ahmadineyad 


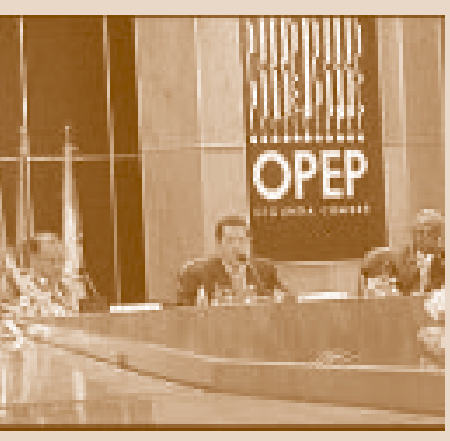

En mayo de 2001, el presidente Chávez visitó Teherán por primera vez, firmando un memorando de entendimiento, que posibilitó la cooperación en los ámbitos económicos, políticos, científicos, tecnológicos y culturales. Por su parte, el entonces presidente iraní, Mohammed Jatami, visitó Venezuela el año 2000 para asistir a la Cumbre de la OPEP y el año 2004, para la celebración de la Cumbre del Grupo de los 15 (G-15). En noviembre de 2004, Jatami realizó una tercera visita a Venezuela, con el fin de consolidar las relaciones bilaterales en el marco económico, firmando acuerdos para la venta a Venezuela de maquinaria y tecnologías en las áreas agroindustrial, agrícola, petroquímica y salud ${ }^{1}$.

Sin embargo, no será hasta la asunción del presidente Ahmadineyad, en junio de 2005, que se afianzarán todavía más las relaciones de Irán con Venezuela y, años después, con otros países de ALBA. Con ocasión de la primera visita del presidente Ahmadineyad a Caracas, también en septiembre de 2006, y luego de su asistencia al Movimiento de los No Alineados, ambos presidentes firmaron 29 acuerdos bilaterales, acordando la construcción de un complejo petroquímico por un valor de 1.500 millones de dólares, una planta automotriz, una fábrica de bicicletas y un fondo de desarrollo conjunto con un costo de 2.000 millones de dólares. Ambos inauguraron un proyecto de exploración petrolera, así como otros proyectos en las áreas de la salud y la agricultura ${ }^{2}$. Irán y Venezuela afianzaron también su alianza energética, con la creación de una empresa para explorar reservas en un bloque de la Faja Petrolífera del Orinoco y el anuncio de un estudio conjunto para construir una refinería en Siria de 150.000 barriles por día ${ }^{3}$.

Al mismo tiempo, durante esta visita, el presidente Chávez se comprometió a apoyar el programa nuclear iraní como parte de la "alianza estratégica” entre ambos países. Según algunas fuentes, este apoyo venezolano no tendría por objetivo sólo contradecir a Estados Unidos y a la Unión Europea, sino que tendría algunos intereses propios: la necesidad de Venezuela de comprar un reactor nuclear para utilizarlo en la exploración petrolera e Irán podría proveer con estos conocimientos nucleares al país sudamericano ${ }^{4}$.

1 "Presidente Jatami llega para consolidar relaciones bilaterales". Gobierno Bolivariano de Venezuela. 10 de marzo de 2005. http://www.mci.gob.ve/noticias_-_prensa/28/7444/presidente _jatami_llega.html (Consulta: 19-01-2009).

${ }^{2}$ Greg Morsbach. "No hay nada que temer", dice Ahmadineyad BBC. 18 de septiembre de 2006. http://news.bbc.co.uk/hi/spanish/latin_america/newsid_5355000/5355208.stm (Consulta: 07-01-2009).

3 "Irán y Venezuela estrechan relaciones energéticas". El M undo. 19 de septiembre de 2006. http:/ /www.elmundo.es/mundodinero/2006/09/19/economia/1158648164.html. (Consulta:27-12-2008).

4 "Interés común: lo nuclear". BBC. 2 de julio de 2007. http://news.bbc.co.uk/hi/spanish/ latin_america/newsid_6262000/6262646.stm\# (Consulta: 27-12-2008). 
Ahora bien, cabe mencionar que el apoyo de Caracas al programa nuclear iraní se produce tres meses antes de que el Consejo de Seguridad de la ONU adoptara la resolución 1737, que impone por unanimidad sanciones a Irán debido a su negativa a suspender su programa nuclear. Anteriormente, en febrero de 2006, Venezuela, Cuba y Siria fueron los únicos países que se opusieron a una resolución del Organismo Internacional de Energía Atómica, que culpó a Irán por violaciones de las salvaguardas nucleares.

Las relaciones estratégicas entre Caracas y Teherán continúan consolidándose durante los años siguientes. Hacia enero de 2007, el presidente iraní, junto con visitar por segunda vez Venezuela, realiza una gira que también incluye Ecuador y Nicaragua, para entrevistarse con sus homólogos, los presidentes Correa y Ortega, ambos aliados de Chávez en la región. Con motivo de esta visita, el mandatario venezolano anunció que ambos países, Venezuela e Irán, crearían un fondo de 2 mil millones de dólares, destinado a ayudar a los países en desarrollo "que aspiran a sacudirse del yugo imperialista"

Por otra parte, en julio de 2007, el presidente Chávez visitó Irán, fortaleciendo todavía más los lazos económicos y estratégicos, al suscribir 17 instrumentos de cooperación en áreas que incluyen conformación de empresas mixtas para procesar productos lácteos y otros alimentos, explotación petrolera, comercio, fabricación de maquinaria pesada, constitución de una empresa mixta para construir 7.000 viviendas en la costa occidental del Lago Maracaibo ${ }^{6}$.

Además, ambos presidentes colocaron la primera piedra de un complejo petroquímico en la zona industrial de Asaluyeh, en Irán, considerado como uno de los más grandes del mundo, con una inversión de 700 millones de dólares y un rendimiento anual de 1,5 millones de toneladas de etanol. Una planta similar está proyectada para ser construida en Venezuela. Ambas plantas permitirían a Irán un mayor acceso a los mercados latinoamericanos, y a Venezuela abrir los mercados de la India y Pakistán. En la visión de Chávez, con este proyecto, Caracas y Teherán buscan "unir el Golfo Pérsico y el Caribe", creando "un mundo multipolar"

\footnotetext{
5 “Irán y Venezuela aúnan esfuerzos para acrecentar la influencia en los países del tercer mundo”. Agencia Rusa de Noticias NOVOSTI. 15 de enero de 2007. http://sp.rian.ru/onlinenews/ 20070115/59101456.html (Consulta: 27-12-2008).

6 "Venezuela e Irán: acuerdos de cooperación”. IADE (Argentina). 5 de julio de 2007. http:/ /www.iade.org.ar/modules/noticias/article.php?storyid=1818 (Consulta: 26-12-2008).

7 "Venezuela e Irán en 'día glorioso". BBC Mundo.com. 2 de julio de 2007. http:// news.bbc.co.uk/hi/spanish/latin_america/newsid_6262000/6262346.stm\# (Consulta: 27-122008).
}

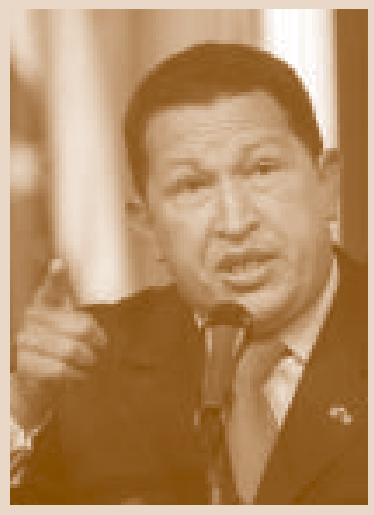

H. Chávez 
En una nueva visita de Chávez a Teherán, en noviembre de 2007, ambos mandatarios, junto con debatir que la OPEP empiece a realizar transacciones en euros en lugar de dólares -siguiendo el ejemplo iraní de cortar todos los lazos con el dólar y, por ende, con Estados Unidos-, acordaron defender en forma conjunta sus intereses y sus ideales y ser portavoces de las "naciones oprimidas y necesitadas". Chávez definió a Irán como su "segundo hogar", "porque los dos estados han establecido una red común política, económica, social y geopolítica que ha alcanzado dimensiones estratégicas" ${ }^{\text {. }}$ Esta visita marcó la undécima vez que Chávez y Ahmadineyad se reunían, la cuarta visita de Chávez a Irán en los dos últimos años y su sexta visita oficial desde que asumió la presidencia en 1999 (Malamud, García, 2007).

Durante el año 2008 se siguió fortaleciendo el eje Caracas-Teherán en los ámbitos financiero y cultural. En el mes de mayo se publicó una ley para la creación de un banco binacional, con los objetivos de crear programas en las áreas de industria, comercio, infraestructura, vivienda, energía y tecnología 9 . En noviembre, la cooperación alcanzó el ámbito cultural, a través de los anuncios de la formación en Venezuela de una Universidad de las Civilizaciones, destinada a fomentar "los principios de la Revolución Bolivariana y del socialismo del siglo XXI". Como parte de este acuerdo, está el intercambio educativo con la formación en la Universidad de Teherán de estudiantes que cursen maestrías y doctorados ${ }^{10}$.

A principios de 2009 se firmó un acuerdo de complementación económica entre ambos países, algunos de cuyos objetivos contemplan: crear "un marco más seguro" para las relaciones comerciales, ampliar el intercambio comercial mediante reducción y eliminación de aranceles, establecer un "comercio justo". Mediante este convenio, los productos venezolanos tendrán el mismo trato que los productos iraníes en el territorio de Irán ${ }^{11}$.

Como parte de esta relación estratégica, hay que mencionar algunas denuncias que señalan que Venezuela estaría violando las sanciones impuestas por la ONU a Irán. Un hecho que es emblemático y que eventualmente

8 "Irán y Venezuela acuerdan defender como unidad sus intereses e ideales". La Jornada (México). 20 de noviembre de 2007. http://www.jornada.unam.mx/2007/11/20/index.php?section $=$ mundo\&article $=032 \mathrm{n} 1 \mathrm{mun}$ (Consulta: 27-12-2008).

9 "Mediante ley crean banco iraní venezolano". 2008. La Guía de Venezuela. http:// www.guia.com.ve/noticias/?id=23141 (Consulta: 21-01-2009).

10 "Irán y Venezuela afianzan lazos con una universidad socialista". ADNMundo.com. 18 de noviembre de 2008. http://www.adnmundo.com/contenidos/politica/iran_venezuela_ afianzan_lazos_con_universidad_socialista_18_11_08_pi.html (Consulta: 27-12-2008).

11“Acuerdo Complementación entre Venezuela e Irán”. Pro-Chile - información estratégica para exportar a Venezuela. 2007.http://www.prochile.cl/ficha_pais/venezuela/relaciones_ internacionales.php (Consulta: 10-01-2009). 
podría tener incidencias en el campo militar es el comienzo de vuelos por parte de las aerolíneas I ran Air de Irán y Conviasa de Venezuela entre Teherán y Caracas, con escala en Damasco, las capitales de tres países que tienen una agenda política contraria a Estados Unidos. Aunque para las autoridades venezolanas e iraníes, estos vuelos son parte de una mayor integración económica y política entre estos países, algunas denuncias apuntan a que estos han servido para ayudar a Irán a transportar material bélico a Siria.

Concretamente, medios de comunicación occidentales, encabezados por el diario italiano La Stampa, citando fuentes de servicios occidentales de inteligencia, denunciaron a partir de diciembre de 2008, que Venezuela utiliza los vuelos de Conviasa para ayudar a Irán a transportar material que sirve para construir misiles a Siria. Entre las empresas involucradas en este intercambio estaría el grupo industrial Shahid Bakeri, empresa afectada por las sanciones de la resolución 1737 del Consejo de Seguridad de la ONU, por "su implicancia en el desarrollo del programa de misiles de Irán". A cambio de esta ayuda venezolana, dicen estas fuentes, Irán habría puesto a disposición de Venezuela a sus Guardianes de la Revolución y miembros de Al Quds para reforzar los servicios secretos y la policía venezolana ${ }^{12}$.

Por otra parte, fuentes también periodísticas han denunciado que autoridades aduaneras del puerto de Mersin en Turquía habrían detectado materiales radiactivos provenientes de Irán y con destino a Venezuela, a través de la empresa mixta venezolana iraní Venirán Tractor. Estas denuncias, así como las que involucran a la línea aérea caribeña, han sido rechazadas por el gobierno venezolano ${ }^{13}$. Estos supuestos lazos militares de Venezuela con Siria e Irán adquieren un matiz importante en la medida que estos dos últimos países, según denuncias israelíes y estadounidenses, apoyan a los movimientos Hezbolá y Hamas, ambos considerados en la lista de agrupaciones terroristas por parte del Departamento de Estado de Estados Unidos y el primero de ellos acusado por la justicia argentina del atentado contra la AMIA realizado en Buenos Aires en $1994^{14}$.

\footnotetext{
12“Venezuela niega transportar material bélico a Siria e Irán.

El diario italiano La Stampa denunció que Venezuela utiliza los vuelos de la línea aérea nacional para ayudar a Irán a transportar a Siria material que sirve para construir misiles". La Tercera. 23 de diciembre de 2008. http://www.latercera.com/contenido/678_87072_9.shtml (Consulta: 23-12-2008).

"Irán evade a ONU por Venezuela". El Universo (Ecuador). 25 de diciembre de 2008.http:// www.eluniverso.com/2008/12/23/1/1361/06E35821B5F34F2D9956885196EFFCA3.html (Consulta: 25-12-2008).

13 "Venezuela acusa campaña que intenta desvirtuar su relación con Irán”. La Tercera. 7 de enero de 2009. http://www.latercera.com/contenido/678_90835_9.shtml (Consulta: 07-01-2009).

${ }^{14}$ Este tema aparecerá en un artículo en preparación, que involucra la participación de Irán y de Hezbolá en los atentados realizados en Buenos Aires en 1992 y 1994.
} 
Cabe señalar que las visitas del presidente iraní a Venezuela han sido motivo de protesta de parte de Estados Unidos, de Israel, de las comunidades judías venezolanas y de todas las comunidades judías latinoamericanas, incluidas las chilenas. El Departamento de Estado de Estados Unidos ha expresado la preocupación de Washington por los nexos de Irán con H ezbolá y con los atentados terroristas llevados a cabo en Buenos Aires en 1992 y 1994. Por su parte, las comunidades judías latinoamericanas y, en particular, la Confederación de Asociaciones Israelitas han rechazado la presencia del presidente iraní en Venezuela por sus amenazas reiteradas al Estado de Israel, así como por la negación del holocausto. A esto hay que sumar el rompimiento de relaciones diplomáticas de Venezuela con Israel, en enero de 2009, y una serie de hechos antisemitas registrados en este país, siendo el más emblemático el asalto que se produjo a una sinagoga de Caracas en febrero de 2009.

En definitiva, los nexos entre Teherán y Caracas van más allá de lo meramente político y económico, constituyen una relación estratégica e ideológica que se enfrenta a Estados Unidos, que es marcadamente antisionista y antiisraelí, que se presenta como defensora de un proceso revolucionario bolivariano en un caso, islamista en el otro-, que se constituye en una alianza que agrupa a otros países -Bolivia, Ecuador, Nicaragua, Cuba- que actúa de forma coordinada en organizaciones internacionales -OPEP, Movimientos de los No Alineados, Grupo de los 15-, y que aparenta tener fines aparentemente militares y nucleares: el apoyo de Caracas y sus aliados al programa nuclear iraní es explícito.

\section{IRÁN Y NICARAGUA: LA REESTRUCTURACIÓN DE UNA VIEJA ALIANZA}

Irán y Nicaragua comparten procesos revolucionarios en forma casi simultánea. La revolución islámica (marzo de 1979), liderada por el ayatola Khomeini, fue seguida de la revolución sandinista (julio de 1979), conducida por Daniel Ortega, ambas con una plataforma que era claramente antiestadounidense. Durante una década -1979-1990- el gobierno de Washington se enfrentó a situaciones revolucionarias simultáneas en Irán y Nicaragua, y a un involucramiento directo en la guerra civil nicaragüense a partir de su apoyo a la denominada "contra" nicaragüense.

En este periodo, y a pesar de la oposición frontal de Washington al nuevo régimen de Teherán, se produce el denominado affaire "Irán-contra", 
maniobra secreta de los servicios de inteligencia de Estados Unidos, encabezados por la CIA, de vender secretamente misiles a Irán, a cambio de la liberación de rehenes estadounidenses mantenidos por Irán. Los excedentes de estas ventas sirvieron para financiar a la Contra. Aunque el presidente Reagan reconoció que tenía conocimiento de los envíos de misiles, negó que se tratara de un intercambio de armas por rehenes. Posteriormente, el Departamento de Justicia reconoció que parte de los beneficios de la venta de armas había sido desviada a la Contra ${ }^{15}$. No obstante este hecho no afectó las relaciones entre Nicaragua e Irán, en un contexto de guerra civil y de presencia militar de Israel, de Estados Unidos y de varios países árabes y musulmanes en Centroamérica (Caro, 1989).

El regreso de Ortega a la presidencia nicaragüense el año 2007 y su alianza con el presidente Chávez son factores importantes que en parte propiciarán un nuevo acercamiento con Teherán. En enero, como parte de su visita a la región, los presidentes Ortega y Ahmadineyad anunciaron la restauración de sus relaciones diplomáticas -las que habían sido rotas en 1990, tras la derrota del sandinismo en las elecciones presidenciales-, reabriendo embajadas en las respectivas capitales. Hay que señalar que este anuncio se produjo cuatro días después de haber asumido Ortega la presidencia nicaragüense, lo que muestra el interés del país centroamericano en mantener relaciones con Irán ${ }^{16}$.

Con motivo de esta visita, ambos mandatarios firmaron un memorando de entendimiento, que contempla la suscripción de acuerdos económicos en áreas que van desde la agricultura hasta el procesamiento de petróleo. En particular, se establece la construcción por parte de Irán de viviendas, represas de desarrollo portuario, comercio de maquinaria agrícola e industrial. También se contempla la creación de fábricas de buses, de cemento e industrialización en el sector del agua ${ }^{17}$.

La visita se dio en un marco de entendimiento político, en que ambos presidentes reiteraron las similitudes de la revolución sandinista y de la islamista, ambas ocurridas el mismo año. En este sentido, como parte de un acto altamente simbólico, Ortega condecoró a Ahmadineyad con la "orden Augusto C. Sandino en su más alto grado Batalla de San Jacinto", batalla

\footnotetext{
15 “La guerra de la Contra y el escándalo Irán-Contra”. 2005. http://www.mayispeakfreely.org/ index.php?gSec=doc\&doc_id=138 (Consulta: 29-01-2009).

16 "Irán y Nicaragua estrecharán lazos". BBC. 15 de enero de 2007.http://news.bbc.co.uk/hi/ spanish/latin_america/newsid_6261000/6261005.stm (Consulta: 07-01-2009).

17 "Ortega y Ahmadinejad firman memorando de entendimiento". 14 de enero de 2007. http://impreso.elnuevodiario.com.ni/2007/01/14/nacionales/38792 (Consulta: 27-01-2009).
}

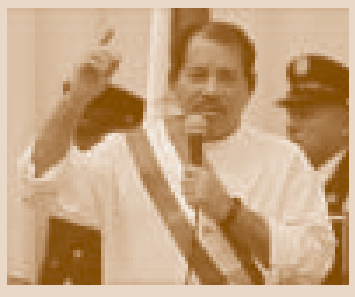

D. Ortega 
que conmemora la lucha de Sandino contra la intervención estadounidense en la década de $1930^{18}$.

En abril de 2007, el presidente Ortega apoyó oficialmente el programa de enriquecimiento de uranio iraní (Malamud, García, 2007). Al mismo tiempo, el ministro de Energía y Minas nicaragüense, Emilio Rappaccioli, anunció el establecimiento de una comisión mixta, destinada a que Nicaragua lograra con Irán un acuerdo para obtener hidrocarburos en condiciones preferenciales, similar a un convenio que ya existe con Venezuela.

Más tarde, en junio de 2007, el presidente Ortega visitó Teherán, se reunió con su par iraní, dio un discurso en la Universidad Islámica de Teherán, donde volvió a señalar que ambos procesos revolucionarios, el sandinista y el islamista, eran gemelos y nacidos el mismo año. Ortega acusó a Estados Unidos, indicando la necesidad de "establecer un nuevo orden mundial y reemplazar el capitalismo y el imperialismo"19. Los dos mandatorios se comprometieron a promover la cooperación política, económica y energética, al tiempo que el presidente Ahmadineyad reiteró la importancia de la cooperación bilateral, con una explícita crítica a Estados Unidos.

Cabe señalar que la embajada de Irán en Nicaragua es una de las más grandes que existen en este país, con un personal que llega a los 40 diplomáticos. Existen denuncias o al menos sospechas de autoridades israelíes, pero no pruebas, de que parte de este personal puede formar parte de la inteligencia iraní y estar asociada con el movimiento H ezbolá que está presente en la triple frontera de Argentina, Paraguay y Brasil, así como en otras zonas sudamericanas ${ }^{20}$.

\section{ECUADOR Y BOLIVIA: DOS NUEVOS SOCIOS ESTRATÉGICOS DE IRÁN}

Los lazos de Irán con Ecuador, al igual que en el caso de Venezuela, tienen un antecedente importante en el marco de la OPEP, donde Ecuador es miembro entre 1973 y 1993, para volver a incorporarse el año 2007, en gran medida como respuesta a la influencia de Irán y Venezuela en esta organiza-

\footnotetext{
18 "Presidente iraní hace planes con Daniel Ortega". 15 de enero de 2007. http://www. telemundo51.com/noticias/10751194/detail.html (Consulta: 27-01-2009).

19 "Ortega de visita en Irán”. BBC. 10 de junio de 2007. http://news.bbc.co.uk/hi/spanish/ latin_america/newsid_6739000/6739657.stm (Consulta: 26-01-2009).

"No es lógica una embajada de Irán en Nicaragua con 40 diplomáticos". La Prensa.

${ }^{20} 6$ de enero de 2009. http://www.laprensagrafica.com/index.php/internacionales/mundo/ 9981.html (Consulta: 26-01-2009).
} 
ción. Sin embargo, a pesar de compartir esta membresía, previo a enero de 2007, son escasos los contactos diplomáticos y comerciales entre Quito y Teherán. En los archivos del Ministerio de Relaciones Exteriores ecuatoriano sólo existe una declaración conjunta previa a 2007, la que fue firmada en 1989 por los ministros de petróleo de ambos países, con motivo de la visita del ministro de Petróleo de Irán a Ecuador (Montúfar, 2008).

La relación bilateral cambia profundamente a partir de la visita que realiza el presidente Ahmadinejad a Ecuador, con motivo de la asunción presidencial de Rafael Correa, en enero de 2007. A partir de entonces, ambos gobiernos abren oficinas comerciales en sus respectivas capitales, se suscriben acuerdos y se realizan visitas mutuas de varias misiones. Estos vínculos no deben verse como un hecho aislado, sino en el marco de una creciente proyección diplomática y de cooperación de Irán con otros países latinoamericanos, que incluyen a Venezuela, Bolivia y Nicaragua (Montúfar, 2008).

El gobierno ecuatoriano, junto con negar que el acercamiento con Irán sea una señal contra Estados Unidos, ha justificado este proceso señalando que se trata de una decisión soberana, manifestando que todos los países de la región mantienen relaciones comerciales con Teherán, y que la iraní es una economía muy complementaria con la ecuatoriana. Al mismo tiempo, ha expresado la posibilidad de establecer un diálogo político ${ }^{21}$. El presidente Correa, al igual que sus homólogos del ALBA, desestimando la participación de Irán en los hechos de la AMIA y la Embajada de Israel en Buenos Aires, ha sido un vehemente defensor de los lazos con Irán, señalando que no le importa lo que piensen otros países "ya que tenemos que ser soberanos y buscar nuestros propios intereses, no tenemos nada contra Irán, no nos ha hecho nada"22.

Sin embargo, la visita del presidente iraní a su homólogo ecuatoriano en enero de 2007 motivó en primer lugar que el presidente argentino, Néstor Kirchner, se negara a asistir a la asunción del presidente Correa, debido a la presencia de Ahmadineyad y a la escasa colaboración que ha prestado Irán en los casos investigados por la justicia argentina con respecto a los atentados contra la AMIA y la Embajada de Israel en Buenos Aires. Por otra parte, el alto representante de la Unión Europea para la Política Exterior, Javier

21 "Ecuador niega que acercamiento con Irán sea una señal contra EEUU”. El Nuevo Diario. 16 de octubre de 2007. http://impreso.elnuevodiario.com.ni/2007/10/16/ultimahora/4927. (Consulta: 07-01-2009).

22“Irán y China están interesados en la nueva refinería ecuatoriana”. M ercosur Noticias. 21 de julio de 2008. http://www.mercosurnoticias.com/index.php?option=com_content\&task =view\&id=11657\&Itemid=34 (Consulta: 11-01-2009).

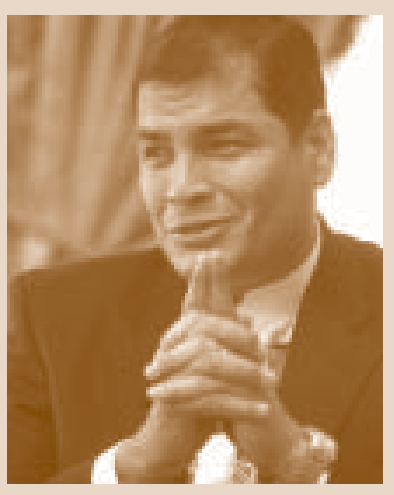

R. Correa

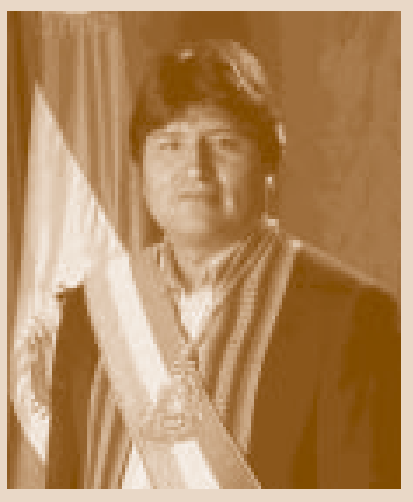

E. Morales 
Solana, ha expresado que Ecuador se está sumando a un "coro latinoamericano" a favor de Teherán, junto a Bolivia, Nicaragua y Venezuela ${ }^{23}$.

En julio de 2008, el presidente Correa informó que Irán, al igual que China, estaba interesado en invertir en una megaplanta petroquímica construida por la petrolera venezolana PDVSA, conjuntamente por Ecuador y Venezuela, en el Pacífico ecuatoriano, con una capacidad para refinar 300.000 barriles diarios de petróleo ${ }^{24}$. En septiembre del mismo año, ambos países firmaron 12 memorandos de entendimiento en las áreas de industria, energía, petróleo, banca, salud, comercio, turismo, tecnología. El presidente ecuatoriano logró la concesión de un crédito de 120 millones de dólares por parte de Irán. En cuanto a la cooperación energética, se concretó lo anunciado a mediados del año, esto es el inicio de la construcción de una refinería y una unidad petroquímica en Ecuador, en conjunto con Venezuela. Este acuerdo permitirá también el entrenamiento a empleados de la industria petrolera ecuatoriana con la ayuda de expertos iraníes ${ }^{25}$.

No obstante, los vínculos entre Ecuador e Irán van más allá del ámbito económico. En diciembre de 2008, en la visita realizada por el presidente Correa a Irán, la primera de un jefe de estado ecuatoriano a este país, Correa calificó de "estratégicas" las relaciones bilaterales, al tiempo que criticó fuertemente a Estados Unidos y favoreció establecer cooperación aduanera y lazos militares con Irán. El presidente Correa, criticando la política exterior de Washington, señaló que el proceso político latinoamericano se construye sobre la base del antiimperialismo, no existiendo lugar para los "radicalismos relacionados con Estados Unidos". Correa se reunió también con el jefe supremo religioso iraní, el ayatola Alí Jamenei, y ambos coincidieron en que los dos países habían escogido "el camino de la resistencia" frente a los poderes globales, encabezados por Estados Unidos.

Por su parte, Correa y Ahmadineyad se comprometieron a establecer relaciones diplomáticas con la apertura respectiva de embajadas a partir de enero de 2009, expandir los vínculos entre ambos estados, aumentar las

23 “Ecuador niega que acercamiento con Irán sea una señal contra EEUU”. El Nuevo Diario. 16 de octubre de 2007. http://impreso.elnuevodiario.com.ni/2007/10/16/ultimahora/4927. (Consulta: 07-01-2009).

24 “Irán y China están interesados en la nueva refinería ecuatoriana”. M ercosur N oticias. 21 de julio de 2008. http://www.mercosurnoticias.com/index.php?option=com_content\&task =view\&id=11657\&Itemid=34 (Consulta: 11-01-2009).

25 “Firman Ecuador e Irán acuerdo de cooperación energética”. La Jornada (México). 15 de septiembre de 2008. http://www.jornada.unam.mx/2008/09/15/index.php?section=economia \&article $=026 \mathrm{n} 1$ eco (Consulta: 27-12-2008). 
alianzas y acuerdos diversos en el marco de la cooperación Sur-Sur ${ }^{26}$. En este sentido, el presidente Correa, acusando al gobierno colombiano de no cuidar su frontera sur, reconoció que Irán podría colaborar suministrando equipo militar, especialmente radares, para salvaguardar la frontera con Colombia.

Por otra parte, cabe señalar que aunque el presidente Correa no ha sido tan explícito como sus homólogos de Venezuela, Bolivia y Nicaragua, en cuanto al apoyo al programa de energía nuclear de Irán, tampoco ha manifestado una oposición explícita al mismo. Correa señaló enfáticamente, en el marco de su visita a Teherán, que no estaba de acuerdo en que "sólo unos pocos países puedan tener energía atómica para razones específicas, incluso para la guerra" 27 .

En cuanto a las relaciones entre Irán y Bolivia, éstas son mucho más recientes. En septiembre de 2007, con motivo del primer viaje del presidente iraní al país altiplánico, ambos estados establecieron relaciones diplomáticas, al tiempo que firmaron un acuerdo marco, de 13 puntos, que incluye inversiones en gas, petroquímica, minería, agricultura, infraestructura, agua, cultura, ciencia y tecnología. El presidente iraní prometió 1.100 millones de dólares en ayuda a Bolivia para los próximos cinco años ${ }^{28}$.

En el ámbito de la cooperación energética, se establecieron actividades en los sectores de hidrocarburos y energía eléctrica, la constitución de empresas de carácter mixto y, quizás lo más importante, la explotación de litio y uranio en la zona de Potosí y Oruro, zonas de alto potencial para la producción de uranio ${ }^{29}$.

Se ha especulado sobre la presencia de uranio en Bolivia y el interés de Irán en este recurso. Por ejemplo, el matutino La Prensa informó en su edición del 26 de septiembre del 2007, que "el gobierno islámico tenía la intención de explorar yacimientos de uranio en Bolivia", lo mismo expresó el periódico estadounidense TheWashington Times el 07 de mayo de 2007. No obstante, el director general de Minería de Bolivia señaló que "no exis-

\footnotetext{
26 “Ecuador, Iran for strategic relations”. 2007. http://www.plenglish.com/article.asp? $\mathrm{ID}=\{$ D5439271-0465-4E94-B31F-8AF8FA65EE52 $\}$ )\&language=EN. (Consulta: 06-01-2009).

${ }^{27}$ Javier de la Fuente. "Los retos de América Latina. El enemigo de mi enemigo es mi amigo. Los lazos entre Irán y Latinoamérica, incómodos para EE UU, se intensifican pero no se traducen en un incremento comercial". El País, Madrid. 15 de diciembre de 2008.

${ }^{28}$ Andrés Oppenheimer. "Claves americanas. El ambiguo interés de Irán”. La Nación. 2 de octubre de 2007. http://www.lanacion.com.ar/nota.asp?nota_id=949217 (Consulta: 08-01-2008).

29 “Ofensiva del presidente iraní en Sudamérica: visita a Evo y a Chávez". Clarín. 27 de septiembre de 2007. http://www.clarin.com/diario/2007/09/27/elmundo/i-02201.htm (Consulta: 0601-2009).
} 
ten yacimientos de uranio en Bolivia, y no hay producción" ${ }^{30}$. Por ahora las actividades estarían en la exploración de este recurso.

Por otra parte, alentado por Venezuela e Irán, y por la decisión tomada por Ecuador, el presidente Morales anunció su intención de ingresar a la OPEP. En una declaración que tiene 13 puntos, ambos mandatarios llaman a promover acciones políticas para fortalecer un "mundo multipolar en aras de garantizar un mayor equilibrio y democratización de las relaciones internacionales" ${ }^{31}$.

En enero de 2009, el presidente Morales visitó por primera vez Teherán, firmando una declaración para la expansión de la cooperación en los ámbitos energético, industrial y comercial. Bolivia, con grandes reservas de gas natural, busca el apoyo de Teherán para desarrollar su sector energético, consiguiendo la promesa del presidente iraní de aportar 1.000 millones de dólares de inversión económica para el desarrollo de la industria de petróleo y gas de Bolivia ${ }^{32}$.

Por otra parte, junto a las condenas de parte de la oposición boliviana -en el sentido de que el interés de Irán era el uranio boliviano-y de autoridades estadounidenses -señalando que esta visita produciría una desestabilización en la región-, el gobierno boliviano dispuso una medida que coincidió con la llegada del mandatario persa: la exigencia de visas para los ciudadanos estadounidenses que quieran ingresar al país.

Parece claro que los vínculos entre Bolivia e Irán, lo mismo que entre Venezuela e Irán, Ecuador e Irán, Nicaragua e Irán, sobrepasan ampliamente el campo de la cooperación económica y política, para situarse en aspectos estratégicos, que en primer lugar dicen relación con el programa de energía nuclear llevado a cabo por el régimen de Teherán. Los presidentes Ortega, Chávez, Morales y Correa, con diversos matices, han respaldado el programa de energía nuclear iraní, subrayando, en el caso del mandatario boliviano, "el derecho de los países al desarrollo de la energía nuclear con fines pacíficos en el marco del Tratado sobre la no proliferación de armas nu-

\footnotetext{
30 "Gobierno boliviano niega que cuente con yacimientos de uranio". Emol. 29 de septiembre de 2007. http://www.emol.com/noticias/internacional/detalle/detallenoticias.asp? idnoticia=277017 (Consulta: 25-01-2009).

31 "Irán recibe apoyo para su programa nuclear 'pacífico". La Razón. 28 de septiembre de 2007.http://www.la-razon.com/versiones/20070928_006043/nota_244_486057.htm (Consulta: 26-01-2009).

32 "Ahmadineyad: Irán y Bolivia son aliados". El Universo. 2 de septiembre de 2008. http:// www.eluniverso.com/2008/09/02/0001/14/ED00D20255EE4288B3A3241A9B32556E.html (Consulta: 28-12-2008).
} 
cleares, como un medio que puede contribuir significativamente al desarrollo económico y tecnológico de sus pueblos" ${ }^{\prime 3}$.

$\mathrm{Al}$ respecto, si revisamos el acuerdo firmado entre Bolivia e Irán, éste menciona el "uso de energía nuclear para fines pacíficos en el marco del Tratado sobre la no proliferación de armas nucleares". Además, firmaron convenios para el desarrollo de actividades en hidrocarburos, minería, producción, industria y otras áreas, por un monto de 1.100 millones de dólares en un período de cinco años.

Sin embargo, las dudas sobre los "fines pacíficos" del programa nuclear iraní son persistentes, al tiempo que estos acuerdos y apoyos o simpatías al programa nuclear iraní se producen en momentos en que los máximos representantes de la Unión Europea, en el seno de la ONU, condenaran firmemente este programa. La canciller de Alemania, Angela Merkel, señalaba que Irán debía convencer al mundo "de que no quiere poseer la bomba atómica", en tanto que el presidente francés, Nicolás Sarkozy, indicaba que si se permitiese a Irán disponer de una bomba atómica "el mundo entero estaría expuesto a un gran riesgo"34.

\section{CONCLUSIONES}

Los lazos mantenidos por Irán con países como Cuba y Nicaragua tienen una data que se remonta hacia fines de la década de 1970, y se enmarcan en los procesos revolucionarios y antiestadounidenses compartidos por las revoluciones cubana, sandinista e islamista. Los vínculos entre Irán y Venezuela, por su parte, tienen antecedentes importantes en el marco de la OPEP, puesto que ambos son miembros fundadores de esta organización petrolera.

A partir de la asunción de Chávez a la presidencia de Venezuela, los nexos con Irán cambian hacia una "relación estratégica", que no sólo cubre aspectos de materia energética, sino que también incorpora elementos destinados a tener una posición común frente a las políticas de Estados Unidos y los conflictos del Medio Oriente. En esta dirección, lo que motiva a ambos

33 “Irán y Bolivia sellaron diversos acuerdos”. La Nación (Argentina) (AFP-ANSA-AP). 27 de septiembre de 2007. http://www.lanacion.com.ar/nota.asp?nota_id=947896 (Consulta: 28-122008).

34 "Asamblea General de la ONU: gran ocasión para la diplomacia”. Deutsche Welle. 25 de septiembre de 2007. http://www.dw-world.de/chinfootball/dw/article/0,,2797303_ page_1,00.html (Consulta: 20-01-2009). 
gobernantes es su posición marcadamente antiestadounidense y antisionista y, en el caso del presidente Chávez, la pretensión de liderar, tanto en América Latina como en el mundo, un movimiento "socialista", "revolucionario" y "bolivariano", que sea contrario a cualquier intento de hegemonía de Washington.

Los aliados de Chávez en la región, representados por Bolivia, Ecuador y Nicaragua, en menor o mayor medida, han seguido los pasos del mandatario bolivariano en sus relaciones con Irán, aumentando los vínculos comerciales, inaugurando relaciones diplomáticas, estableciendo cooperación energética, promoviendo las visitas presidenciales bilaterales, manteniendo una agenda en común que busca hacer frente a la presencia estadounidense y apoyando, con diversos matices, los planes nucleares de Irán.

Estas relaciones adquieren especial preocupación para Estados Unidos, para Israel, para la Unión Europea y para las comunidades judías de todo el mundo, por dos razones principales. Primero, Irán ha llevado a cabo un programa de enriquecimiento de uranio, que ha sido condenado en el marco de la Organización de Naciones Unidas, pero que sin embargo ha sido defendido por los miembros fundadores del ALBA. Segundo, Irán y el movimiento pro iraní Hezbolá, han sido responsabilizados de los ataques realizados en Buenos Aires en contra de la Embajada de Israel (1992) y en contra de la AMIA (1994).

Adicionalmente, la presencia de Irán está relacionada con aspectos militares y estratégicos, cuyas consecuencias, tanto para el Medio Oriente como para la región latinoamericana, podrían ir en tres direcciones. En primer lugar, los yacimientos de uranio que existen en esta parte del mundo podrían eventualmente servir a Irán para promover su programa de energía nuclear más allá de fines meramente pacíficos. En este sentido, puede explicarse en parte la importancia que Teherán ha dado a la suscripción de acuerdos de cooperación en este ámbito.

En segundo lugar, el establecimiento de vínculos militares -como la presencia de radares y equipo militar iraní en la frontera de Ecuador con Colombia o la eventual participación de Guardianes de la Revolución Iraní en la policía venezolana- puede ser un detonante de conflictos entre países sudamericanos que comparten visiones opuestas tanto en el campo ideológico como en el de la seguridad militar y regional. Estos lazos en el ámbito militar son todavía más significativos dado el quiebre de relaciones diplomáticas entre Ecuador y Colombia, las volátiles relaciones entre Venezuela y Colombia, y la presencia de la FARC en un territorio que sobrepasa los límites colombianos. 
En tercer lugar, la presencia de Irán en América Latina ha implicado, de un modo u otro, importar los conflictos del Medio Oriente a través de una posición compartida por países del ALBA, que es marcadamente antiestadounidense, antiisraelí y antisionista. En este sentido, cabe mencionar los múltiples incidentes antisemitas registrados en Venezuela, así como el rompimiento de relaciones diplomáticas de Bolivia y Venezuela -dos de los aliados regionales de Irán- con Israel en enero de 2009, por el conflicto entre Israel y Gaza.

\section{REFERENCIAS}

Arjomand, Said Amir. 1986. “Iran's Islamic Revolution in comparative perspective", World Politics, USA, Vol. 38, № 3, April, pp. 383-410.

Caro, Isaac. 1989. América Latina en el mundo militar. Santiago: FLACSO, Salesianos.

Malamud, Carlos, García Encina, Carlota. 2007. Los actores extrarregionales en América Latina: Irán. Madrid: Real Instituto Elcano, ARI No 124.

Montúfar, César. 2008. "El reciente acercamiento diplomático entre Ecuador e Irán ¿Gesto de afirmación soberana o tibio alineamiento geopolítico?”, Conference: I ran in Latin America: Treta or Axis of Annoyance, Woodrow Wilson International Center for Scholars.

Romero, Carlos. 2006. "Venezuela y Estados Unidos: ¿Una relación esquizofrénica?". Nueva Sociedad 206, pp. 78-93.

Torres Soriano, Manuel R. 2006. "La fascinación por el éxito: el caso de Hezbollah en América Latina". Jihad M onitor O casional Paper No 1. Sevilla: Universidad Pablo de Olavide de Sevilla. 